\title{
Cardiac failure in $\beta$-thalassemia: diagnosis, prevention and management
}

\author{
A. Aessopos ${ }^{1}$, D. Farmakis', V. Berdoukas² \\ ${ }^{1}$ First Dept. of Internal Medicine, University of Athens Medical School, Laiko Hospital, Athens, \\ Greece; 'Division of Haematology/Oncology, Children's Hospital, Los Angeles, CA, USA
}

\begin{abstract}
Heart failure always represented and still remains the leading cause of mortality in $\beta(\beta)$-thalassemia, despite the therapeutic advances and the considerable amelioration of prognosis accomplished over the last decades. High cardiac output due to chronic anemia and myocardial iron overload due to repetitive blood transfusions are the two main pathogenetic mechanisms causing heart failure in $\beta$-thalassemia. In regularly treated thalassemia major patients, left ventricular dysfunction, resulting mainly from myocardial siderosis, is considered to be the primary cause of heart failure and thus the prevention, early recognition and effective management of iron overload is of key importance. However, the spectrum of cardiovascular complications that may ultimately lead to heart is wide and should be individually investigated in each one of the patients. Echocardiography is the main modality used for the regular follow-up and screening of asymptomatic patients and for the evaluation of patients with cardiac symptoms, while the T2* relaxation time provided by magnetic resonance imaging allows the accurate identification and quantification of myocardial iron burden and thus the proper guidance of iron chelation therapy.
\end{abstract}

\section{Introduction}

Patients with the major form of homozygous $\beta$ thalassaemia require regular blood transfusions for survival. Without transfusions death usually occurred within the first decade. Subsequently with adequate transfusions patients grew well and survived well until the second decade when complication from iron overload would lead to pre mature

Correspondence: A. Aessopos, First Dept. of Internal Medicine, University of Athens Medical School, Laiko Hospital, Athens, Greece.

Key words: thalassemia major, thalassemia intermedia, heart failure, iron overload, iron chelation, cardiovascular magnetic resonance, $\mathrm{T} 2 *$.

(C) Copyright A. Aessopos et al., 2011

Licensee PAGEPress, Italy

Thalassemia Reports 2011; 1(s2):e16

doi:10.4081/thal.2011.s2.e16

This article is distributed under the terms of the Creative Commons Attribution Noncommercial License (by-nc 3.0) which permits any noncommercial use, distribution, and reproduction in any medium, provided the original author(s) and source are credited.

Parts of this work were presented at the "12th International Conference on Thalassemia and Hemoglobinopathies”, Antalya (Turkey), 11-14 May 2011. death particularly because of heart disease. Iron chelation therapy has significantly improved the survival even more and reduced the morbidity. ${ }^{1,2,3}$ However, heart complications still represent significant morbidity and remain the leading cause of mortality in transfusion dependent thalassaemia (TM) patients. ${ }^{4}$ Cardiac dysfunction with congestive cardiac failure (CCF), arrhythmias still lead to premature death. $5,6,7$

\section{Pathophysiology}

Cardiac structure and function in TM are mainly affected by two factors: increased cardiac output and iron load.

\section{Increased cardiac output}

Anaemia together with marrow expansion leads to volume overload that then demands increased contractility. (Starling's Law). Disease related increased $\mathrm{CO}$, resulting in increased workload on the heart, contributes to the development of cardiac dysfunction in TM patients. In normal individuals, $\mathrm{Hb}$ levels between $8-10 \mathrm{~g} / \mathrm{dl}$ do not have any effect on the cardiac output. ${ }^{8,} 9$ TM patients, however, even those well transfused ( pre transfusion $\mathrm{Hb}$ level $>9.5 \mathrm{gr} / \mathrm{dl}$ ) with excellent suppression of marrow activity and with mean $\mathrm{Hb}$ level between transfusions $11.3 \mathrm{gr} / \mathrm{dL}$, demonstrate some degree of high cardiac output (Cardiac Index $4.3 \pm 0.9 / 3$.in TM cf. 3.8 $\pm 0.8 \mathrm{P}<.01$ in normal individuals. It is more obvious in cases were low Hb levels and tissue hypoxia stimulate compensatory reactions leading to development of peripheral shunts. ${ }^{10}$ Liver iron load or viral induced hepatic injury can also contribute, as cirrhosis can increase CO significantly. ${ }^{11}$ Furthermore, the presence of elastic fibre degeneration, affecting elastic lamina and adventitia, which render vessels more susceptible to dilatation by pulse pressure increase in the context of a hyperkinetic state also increases the total blood volume. ${ }^{12}$

In patients with high output state, the heart's systolic function index and ejection fraction is expected to be higher than in normal subjects. Thus, for TM patients, even in well transfused, it has been recommended that a normal LVEF should be above $60 \% 13,14$ and the degree of $\mathrm{CO}$ increase should be taken into account when assessing EF in each individual patient. ${ }^{15}$

\section{Iron overload}

Iron overload results principally from the regular blood transfusions. Patients receive between $0.3-0.5 \mathrm{mg} / \mathrm{Kg} /$ day of iron through transfusions. The average daily losses are less than $1 \mathrm{mg}$ in males and $2 \mathrm{mgs}$ in menstruating females. There are no other physiological mechanisms effecting body iron reduction. In addition to the transfused iron, TM patients absorb more iron than normal individuals, most likely due to a paradoxical suppression of hepcidin. ${ }^{16,17,18}$

The accumulated iron is thought to saturate liver firstly, and then to 
accumulate in other organs. In heart, it accumulates in all four chambers, papillary muscles and the electrical conduction system, including the sinoatrial and atrioventricular nodes. In the free wall of the left ventricle there is more iron concentrated in the epicardial layers than in the endocardial and middle third.

Iron is stored in cells, including myocytes, in the form of ferritin, haemosiderin and free iron. The latter is referred as the labile cellular iron (LCI). ${ }^{19}$ There is a significant flux between the three forms, with haemosiderin being the least accessible. The LCI is the most toxic form as it stimulates the formation of free radicals, which results in peroxidative damage of membrane lipids and proteins provoking cellular injury. In heart, this leads to impaired function of the mitochondrial respiratory chain and is clinically manifested by reduction of cardiac muscular contractility and CCF development. ${ }^{20}$ Histology has shown individual myocyte hypertrophy with multiple deposits of brown granular material within the cytoplasm of the myocytes. ${ }^{21}$ Moreover, the study of cardiac biopsies from TM patients with light and electron microscopy, as well as with X-ray microanalysis has revealed the presence of disrupted myocytes showing loss of myofibers, dense nuclei, and a variable number of pleomorphic electron dense granules. These cytoplasmic granules or siderosomes consist of iron-containing particles as confirmed by X-ray microanalysis.

To date, at least 90 genes that control iron metabolism have been identified. ${ }^{22}$ In each individual therefore, it is highly likely that the handling of iron and the action of iron chelators will be different. These concepts fit in well with the wide range of reported different clinical cardiac courses seen in TM patients who have followed similar lifetime, well accepted treatment. ${ }^{6}$

Knowledge derived by recent MRI studies which also assessed cardiac function, showed that all patients with reduced LV function had cardiac iron overload and in many cases this was severe. ${ }^{23,24,25,26}$ This strongly suggests that in addition to the damage caused by the accumulated iron, excessive iron in the myocytes results in greater amounts of LCI leading to free radical formation that overwhelms the antioxidant mechanisms and ultimately precipitates cardiac dysfunction. On the other hand, in the above MRI studies, despite heavy iron load, many TM patients maintained normal cardiac function, albeit perhaps temporarily, and, as discussed above, this may be related to their intracellular iron metabolism, in particular their handling of oxidants. It has been shown that TM individuals who had the genetic factor apo-lipoprotein $\mathrm{E} 4$ are at greater risk for $\mathrm{LV}$ dysfunction than those with other alleles as apoE4 compared to apoE2 and apoE3 is less efficient in handling oxidative stress. ${ }^{27,} 28$

The iron induced cardiac toxicity is often complicated by arrhythmias such as extra atrial and ventricular beats, paroxysmal atrial tachycardia, flutter or fibrillation and rarely ventricular tachycardia. Some of these can also be triggering factors for CCF or reduced cardiac function in TM patients without previous obvious LV dysfunction.

Patients with heart failure and cardiac siderosis, as determined by Cardiac MRI, when managed with intensified chelation have improved their cardiac function and reduced the cardiac iron. ${ }^{29,} 30$ Improvement in cardiac function is more rapid than the relative clearance of iron, ${ }^{31}$ while the liver clears much more rapidly that the heart. ${ }^{32}$

Besides direct myocardial injury, iron overload also causes indirect cardiac effects through its extra-cardiac complications, including infections, vascular disorders, other organ damage and related medications.

Any significant infections may precipitate cardiac failure particularly in the presence of other underlying cardiac pathology. Immune competence in $\beta$-thalassemia is impaired. ${ }^{33,34,35,36}$ Iron overload is considered to be the main etiologic factor that can disturb the immune balance in favour of the growth of infectious organisms. ${ }^{37}$ Differences in the existing immunogenetic profile in TM may also play a role. ${ }^{38}$ In addition, some siderophore bacteria such as yersinia and klebsiella, rely on iron for multiplication and grow well in the microenvironment of TM patients. ${ }^{35,39}$

Two severe cardiac complications are linked to iron load induced viral infections susceptibility. Pericarditis, frequently seen (50\%) in TM patients with poor or no chelation in the past, ${ }^{40}$ is very rare today (5\%), with the use of chelation therapy. ${ }^{6}$ Similarly, the reported myocarditis in TM patient ${ }^{41}$ with decreased LV function must be related to iron load. Even though there may be histological evidence of infectious myocarditis, LV failure only occurs in the presence of excessive iron. ${ }^{23,24,26}$ It is unlikely that viral infection alone would be responsible for decreased LV function. On the other hand the selective heart iron deposition in the muscular epicardium encroaches upon the pericardium. Such iron deposition raises a possibility that pericarditis myocarditis may also have an iron induced chemical inflammatory component and also may cause fibrosis of the pericardium with or without a history of pericarditis.

Systemic arterial involvement plays a role in the development of cardiac dysfunction as observed recently through clinical, functional ${ }^{42,43}$ and anatomical ${ }^{12}$ studies. Vascular involvement starts early in life and becomes obvious in the older patients. ${ }^{44}$ The anatomical component including elastic tissue abnormalities is expressed in arteries by thickening and disruption of the elastic laminae and adventitia, followed by calcium deposition. Arterial functional abnormalities are improved by intensive combined chelation therapy ${ }^{32}$ or by the administration of deferasirox monotherapy. ${ }^{45}$ The injury is thought to be mediated by the increased labile plasma iron (LPI), along with the chronic haemolytic state. Erythrocyte membrane fragments, haem and free haemoglobin in addition to free iron, provoke a strong oxidative stress on endothelium. ${ }^{44} \mathrm{~A}$ component of the vascular dysfunction is due to the reduction of NO. There are at least three mechanisms responsible for that reduction either by reducing the bioavailability of NO, by reducing its production or by neutralising it. The oxidative stress inactivates endothelial cells enzymes and reduces formation of NO from the precursor arginine. Furthermore haemolysis releases arginase which reduces arginine levels and supplementation to the endothelium, while oxyhaemoglobin reacting with $\mathrm{NO}$ and converting it to inactive $\mathrm{NO}^{3}$, is transformed to methhaemoglobin. ${ }^{46}$

Similar mechanisms apply also to the pulmonary artery and its vascular contribution together with coexisting hypercoaguability is considered to be responsible for increased pulmonary artery resistance. ${ }^{47,48}$

In addition, iron toxicity may also indirectly affect heart function by the varying degrees of other organ injury and functional impairment. Endocrine abnormalities dominated by the frequently present hypothyroidism and diabetes mellitus as well as the infrequent presence of overt hypoparathyroidism have a significant impact on cardiac function. ${ }^{49}$ Hypocalcaemia can precipitate heart dysfunction.

Vitamin $\mathrm{C}$ has been given to patients in order to enhance their iron excretion when they are on chelation therapy. There have been case reports of patients who developed sudden acute cardiac failure with a fatal outcome that had been precipitated by the administration of Vitamin C possibly by releasing free iron that is toxic. ${ }^{50}$

Summarizing the mechanisms of injury in TM, the iron overloaded heart, is obliged to maintain a high output through a rigid vascular bed, resulting from the abovementioned vascular damage and therefore a continuous state of both volume and pressure overload renders the LV more susceptible to decompensation.

Similarly, in TM patients the coexistence of high cardiac output state and gradually increasing pulmonary vascular resistance (PVR) seems to lead to the development of pulmonary hypertension (PHT), which readily precipitates $\mathrm{RV}$ failure. ${ }^{48}$ In well-treated TM patients, the inhibition of the above mechanisms, result in a considerable reduction of LV and vascular dysfunction, ${ }^{32}$ pulmonary hypertension development and RV failure. ${ }^{51}$ 


\section{Cardiac manifestations}

In TM patients, due to the different abovementioned mechanisms and their degree of contribution to the cardiac damage, including the different treatment regimes followed, as well as the presence or absence of pericarditis with or without myocarditis, genetic factors and endocrine abnormalities, the heart involvement is the result of a mixed action of all those interfering factors. Echo Doppler study offers the ability to identify cardiac pathology. However, it should be kept in mind that all anatomical and functional systolic and diastolic indices are modified by diverse action of the contributing factors and cannot be completely compared to normal individuals. ${ }^{6,8,14}$ Nowadays in TM patients, CCF from high $\mathrm{CO}$ alone, as a result of inadequate transfusions is rare. Similarly, restrictive type cardiomyopathy findings resembling constrictive pericarditis, which are also seen in patients with hemochromatosis, ${ }^{52,53}$ are uncommon today in CCF in TM patients. The usual findings in CCF TM patients are dilated cardiomyopathy with biventricular dilatation and decreased LV and RV systolic function. This is always associated with diastolic abnormalities on the Doppler study, that usually precede systolic impairmentvi. ${ }^{6}$ Some patients, mainly with thick pericardium may develop decreased LV function without obvious ventricular dilatation. It should be stressed thought that also pericarditis is rare nowadays. Severe hypocalcaemia, hypothyroidism, hypoparathyroidism and diabetes mellitus further complicate the clinical picture. The development of PHT may accompany the CCF in almost all the above forms and is more likely to precipitate right-sided heart failure; however, severe PHT is rare in optimally treated patients. In cases with impaired LV function, thrombus formation in the apex of the heart may be present and can lead to the development of stroke. ${ }^{54}$

\section{Clinical presentation}

Cardiac involvement can present clinically with heart failure, arrhythmias and pericarditis. Heart failure can present at any time after the age of ten years but with optimal treatment, heart failure usually occurs not earlier than the third or fourth decade of life. ${ }^{6}$

Although some patients can present with symptoms of left-sided heart failure including exertional dyspnea, cough and fatigue, followed by rales and gallop rhythm on chest auscultation, it is worth noting that the majority presents with symptoms and signs of right ventricular dysfunction. The patients often present to an outpatient clinic with severe fatigability and abdominal pain, the latter due to liver distention. The patient may be lying on the examination couch without dyspnoea. These signs can easily be misinterpreted as not being symptoms of cardiac origin. 55,56

However, clinical examination with the patient in a correct position, will reveal a positive hepatojugular reflux with neck vein distention. In addition, a third heart sound, peripheral edema and ascites may be found. Chest X-ray shows cardiomegaly but frequently there are no features of pulmonary congestion. Echocardiogram usually shows biventricular dilatation and reduction of function. This peculiar clinical appearance in TM patients should be kept in mind. It results from the thin iron loaded right ventricle that decompensates earlier. ${ }^{57}$ In this young population, it has often been associated with a gradual reduction in physical activity, which obscures and delays the presentation.

The presentation of pericarditis is similar to that which occurs in the general population. This is also the case with the arrhythmias. However, some arrhythmias may precipitate CCF.

\section{Management}

TM patients with signs and symptoms of CCF should be hospitalized and closely monitored. Extensive laboratory tests should be done including arterial blood gas, endocrine profile as well as liver and renal function tests. Chest $\mathrm{x}$-ray, apart from cardiomegaly, lung congestion and pleural effusion, may show a prominent pulmonary artery in cases with coexisting pulmonary hypertension. It is unusual for the ECG to be normal. Wide QRS complex with low voltage, inverted T waves, non specific ST-T changes, Left Ventricular Hypertrophy (LVH), prolonged A-V conduction and arrhythmias are frequently seen. Doppler echocardiographic study should also be performed in order to determine the severity of left and right ventricular systolic and diastolic dysfunction, whether there is pericardial involvement and whether pulmonary hypertension is present. As stated above recent MRI studies have confirmed that almost all patients with decreased left ventricular function have severe iron load. ${ }^{23,} 25$ In those cases the values of MRI measurements are only important to determine the degree of iron overload for future follow-up and can be postponed till after the patient has improved clinically.

Triggering factors for CCF development such as arrhythmias, blood volume overload after transfusion, infections, severe anemia, should be identified and treated. In cases where Yersinia enterocolitica or Klebsiella pneumoniae infection is suspected ${ }^{58}$ patients should be treated even before immunological or bacterial culture test results are available. If arrhythmias are present, the least negative inotropic antiarrhythmic agent, amiodarone should be infused intravenously. ${ }^{59}$

Daily measurements of body weight, blood pressure and 24-hours urine secretion are of paramount importance in these patients. Frequent monitoring of Hematocrit, Hb, blood electrolytes, urea, creatinine, glucose, AST, ALT, uric acid, is also mandatory.

\section{Chelation therapy}

Chelation therapy protects against the development of CCF. It is thought that LPI and LCI are very accessible to chelation therapy and at least LPI that can be measured is rapidly reduced in the presence of any of the three available chelators. The rapid improvement in cardiac failure often seen with the intensification of chelation therapy is thought to be related to this latter mechanism. Combination of the two iron chelators (deferrioxamine and deferiprone) seems to maximize the efficacy producing additive and synergistic effects in iron excretion. ${ }^{60}$, ${ }^{61}$ It seems that each of those two agents chelates iron from different pools and there is at least an additive effect when combined treatment is administered ${ }^{62}$ Available evidence now suggests that combined therapy should be the treatment of choice for patients with established cardiac failure. We have reported two cases with severe CCF who reversed with intensive combination therapy ${ }^{29,49,63}$ and we have at least 8 more patients with similar outcome. Two other studies show similar responses. ${ }^{30,61}$ In a recent study combined treatment, in addition to significant reduction in ferritin, cardiac and live iron and improvement in cardiac function, with respect to vascular function, the absolute endothelial function was also improved. ${ }^{32}$ There has even been improvement with some endocrinopathies with combination therapy. ${ }^{64}$ Desferrioxamine should be administrated at a dose of $60-80 \mathrm{mg} / \mathrm{kg} /$ day, best over 24 hours, intravenously and deferiprone at a dose of $75-100 \mathrm{mg} / \mathrm{kg} /$ day in three divided doses. If deferiprone is contraindicated, the patient should be managed with continuous desferrrioxamine infusions, which usually requires the placement of an indwelling catheter. ${ }^{65}$

Caution should be taken with the $24 \mathrm{~h}$ desferrioxamine infusion to avoid fluid overload especially when intravenous antibiotics and antiarrthymic agents are also indicated. 


\section{Blood transfusions}

Since cardiac output may be close to normal when Hb level is $\geq 10 \mathrm{~g} / \mathrm{dL}$, patients' $\mathrm{Hb}$ concentration should be kept above $10 \mathrm{~g} / \mathrm{dL}$ by regular blood transfusions. The patients should only be transfused with one packed red cell unit no greater than $250 \mathrm{ml}$ and diuretic treatment as well.

\section{Endocrine abnormalities}

If hypothyroidism is present, a titrated dose of hormone replacement should be carefully initiated. Diabetes mellitus should be regulated with caution. Although hypoparathyroidism is generally rare in thalassemic population, it is more often seen in patients with congestive cardiac failure and shows the typical ECG abnormalities of prolonged QT interval. ${ }^{66}$ Calcium is necessary for heart muscle cell contractility. CCF with the coexistence of low serum calcium levels is extremely resistant to conventional treatment. ${ }^{67}$ Serum calcium level should be corrected by intravenous calcium administration accompanied by oral vitamin D.

\section{Cardioactive medications}

Diuretics, including loop diuretics (e.g. furosemide) and potassiumsparing agents (e.g. spironolactone), as well as ACE-inhibitors should be prescribed based on arterial blood pressure. In cases of persistent normal sinus tachycardia, small doses of Carvedilol may be given. Digoxin must be prescribed to patients with atrial fibrillation resistant to conversion. Diuretics must be prescribed with care for reasons stated in the following section.

\section{Additional modalities}

If pulmonary hypertension is present, then based on the recent observation of reduction of $\mathrm{NO}$ availability in hemolytic anemia, sildenafil which releases NO, or other medications for pulmonary hypertension, should be given with care. ${ }^{47}$ Hypoalbuminemia is often present. The combination of excessive hepatic iron, hepatic viral infections and other recent infections with the stress of congestion leads to liver dysfunction, which, in turn, results in reduced albumin production. The low serum albumin level could be masked by diuretic administration. It is important to note that TM patients with CCF, after strong diuresis, in combination with hypoalbuminemia are at significant risk of developing reduced renal function or acute renal failure caused by further reduction in cardiac output due to significant preload reduction in the presence of ventricular dysfunction. Thus, careful albumin administration with a gradual negative fluid balance by diuretics is necessary. In case of reduction of renal output, treatment with intravenous positive inotropic agents (e.g. dopamine -dobutamine) in a titrated dose alone and/or with hemodialysis should be considered.

The above treatment can yield very positive results, often within a short period of time - even before any significant reduction in the cardiac iron load would be expected. If the patient survives the acute phases of CCF, treatment should be continued. Patients' general clinical condition, echocardiographic studies and MRI T2* measurements could guide subsequent treatment modifications. Until the ejection fraction approaches normal, transfusions should be given at 7-10 daily intervals with reduced amounts of packed cells avoiding volume overload. In particular, changing the intravenous deferoxamine to daily subcutaneous infusions once the patient is stabilized, is the first step. This allows the patient to be discharged from hospital. Ultimately, heart function may revert to normal class I (NYHA).

\section{Prevention}

Ideally, to prevent body iron accumulation, chelation treatment should lead to, at least, equilibration between iron received through transfusions and iron excretion. However, the delay in commencing iron chelation, the agents toxicities, the compliance to the chelation, the individualization of iron deposition in different organs and the different chelation agent action, together with the difficulty of iron excretion measurements make the overall assessment of the iron balance difficult. Furthermore, all the tests such as ferritin levels, ${ }^{31,68}$ liver iron concentration ${ }^{69,70}$ and conventional assessments of cardiac function with Chest Radiography, ECG and echocardiography ${ }^{71}$ have limited use in predicting the patients cardiac risk. Cardiac dysfunction and death occurred even in patients who accepted conventional chelation and transfusion therapy very well. Magnetic resonance imaging techniques (MRI) have revolutionized the approach, at least by identifying patients at risk, giving the ability to modify the management of TM and to prevent heart disease. A number of studies have demonstrated the value of Cardiac Magnetic Resonance Imaging (CMR) in indirect assessment of cardiac iron overload (T2*) and function parameters. ${ }^{23,72,73,74,75}$ Many other centres are instituting either the same or similar MRI techniques. The results appear to be comparable using different machines and in different countries. ${ }^{76}$ They are reproducible and robust, provided the $\mathrm{T} 2 *$ method is used and the area measured is the intraventricular septum. ${ }^{77}$ The classification of patients is that those with $\mathrm{T}^{*}$ between 20-25 mss are regarded as not having cardiac iron. Those with $\mathrm{T} 2 *$ between $10-20 \mathrm{~ms}$ have mild to moderate cardiac iron load and those $<10$ mss are considered to have heavy cardiac iron load. ${ }^{23,24,25}$

The major benefit from the use of MRI is that it allows the comparison of iron load to heart function. It is clear that cardiac damage is related to the amount of cardiac iron. MRI provides the ability to determine the predictive value of ferritin, LIC ,echo and BNP with respect to cardiac iron as well as that of the patients' compliance to therapy and their red cell consumption. Furthermore with the confidence in the studies, we can make appropriate treatment modifications, for each individual patient based on the assessed cardiac iron load and finally it allows monitoring of the effect of the treatment modifications, thereby preventing the onset of cardiac dysfunction. ${ }^{65}$

The disadvantages of CMR are that it is expensive, time consuming, performed within a claustrophobic environment, and cannot be used in patients with cardiac pacemakers or metallic implants in their upper torso. Also, it is not universally available.

Heart iron MRI measurements are limited to the deposited forms of ferritin and haemosiderin that comprises the majority of the cellular iron but it cannot measure the toxic labile cellular iron (LCI). Despite this, given the fact that a continuous interaction between the three forms of iron exists, MRI measurement remains the best method for estimating potential iron toxicity.

\section{Chelation therapy}

Chelation treatment today should be guided by MRI findings, if the technique is available. We are in a transient phase of knowledge with the availability of MRI and new chelating agents. Important questions with respect to best management to avoid iron induced cardiac disease remain to be elucidated. Optimal management may be clarified from results of different trials and current ongoing follow up studies from many subgroups of patients using different regimes.

In the presence of excess cardiac and or hepatic iron, treatment strategies include increase of the dose and/or frequency of desferrioxamine, switch to oral chelators (deferiprone or deferasirox) or to the combination of deferiprone with desferrioxamine, provided there are no contraindications to their use. ${ }^{32}$ The efficacy of the two oral chela- 
tors is at least equal to the standard doses of desferrioxamine with respect to hepatic iron removal. Recent and ongoing studies have demonstrated that deferiprone, a small molecule that permeates all tissues, is more efficient in removing cardiac iron and improving cardiac function than desferrioxamine ${ }^{78,79}$ Some preliminary clinical and laboratory observations with deferasirox are encouraging with respect to removal of cardiac iron..$^{20,80,81}$ As yet, there are no studies with combinations of deferasirox and desferrioxamine so this therapeutic regime cannot be recommended at this stage.

According to the current knowledge and based on the CMR findings, the suggested chelation regimes are as follows.

For patients with cardiac $\mathrm{T} 2 *$ greater than $20 \mathrm{~ms}$., the therapeutic strategy should be continuation of monotherapy with either desferrioxamine or either of the available oral chelators (deferiprone and desferasirox). For patients' convenience, desferrioxamine administration may be converted to either of the two oral chelators.

$\mathrm{T} 2 *$ values between $10-20 \mathrm{~ms}$ are considered to reflect a mild to moderately iron loaded myocardium. A reasonable suggestion could be that patients with $\mathrm{T} 2 *$ values close to relatively normal values (T2* between $15-20 \mathrm{~ms}$.) could be treated with monotherapy of either deferiprone ${ }^{78,79}$ or deferasirox together with careful follow up (i.e if the latter, MRI assessment at 6 months until prospective data are available). Patients treated up to the time of the MRI with desferrioxamine in this category and who availed themselves of that treatment satisfactorily, should not be on monotherapy with that compound, as deferrioxamine was inadequate at preventing the iron accumulation in the heart and may indicate some type of resistance to its efficacy within that patient. On the other hand, bearing in mind that the patients with mild iron load, may be at risk of developing cardiac problems under stress such as infections or pregnancy, clearing myocardial tissue from iron seems to be a rational target. Therefore, combined treatment for these patients should not be a priori excluded.

Patients have presented with LV dysfunction at levels of T2* of 15 $\mathrm{ms}$, without any precipitating factors. ${ }^{25}$ Therefore, if T2* is $10-15 \mathrm{~ms}$, combination chelation therapy is recommended. However, questions still exist, regarding the frequency and the amount of desferrioxamine administration that is appropriate in a combined regimen. A dose of 35$40 \mathrm{mg} / \mathrm{kg} /$ day three-four times weekly combined with deferiprone at a dose of $75 \mathrm{mg} / \mathrm{kg} /$ day seems to be reasonable.

Patients with $\mathrm{T} 2 *<10 \mathrm{~ms}$ are considered to have severe iron overload and this category includes most patients with reduced left ventricular (LV) function. Even those patients with normal ejection fraction in this category are considered to be at risk for cardiac dysfunction. Thus all patients in this category have a strong indication for combined chelation treatment. The doses of the two medications should be similar to those described for patients with CCF but with the desferrioxamine administered subcutaneously.

Any treatment modification should be followed by close monitoring. Should any serious adverse effect present as a consequence of the administration of a particular chelator, appropriate guidelines as to its continued use should be followed. Patients should undergo MRI study every 1-2 years in order to estimate the long term efficiency on iron removing from heart and liver.

If treatment has modified the MRI patient's classification then, it may be adjusted as discussed above according to the changes in MRI values. In addition to heart iron load estimation, chelation treatment adjustment should also take in consideration other organs iron load particularly liver.

In countries where MRI is not available, then all the patients' traditional parameters need to be analysed, (ferritins, liver iron concentrations) as well as ECG and echocardiogram, taking into account the previously discussed limitations. These may serve as a guide to treatment. Furthermore, according to knowledge from MRI studies in countries where follow up of patients occurs, In Sardinia, up to $65 \%$ of patients have cardiac iron load and $13 \%$ had severe cardiac iron overload. ${ }^{26}$ In our initial study $48 \%$ of patients receiving monotherapy with desferrioxamine with normal cardiac function had cardiac $\mathrm{T} 2 *<15 \mathrm{~ms}^{25} \mathrm{In}$ countries were patients compliance to treatment is inadequate, there was poor availability of chelation and/or the follow up was not well organized, the percentage of cardiac iron loaded patients is likely to be higher. Therefore, for patients who have never had optimal care, it is very likely the patients will have cardiac iron load and intensive chelation is the treatment of choice. In patients who have been poorly chelated, the risk of chelation toxicity is minimal and would only be likely to occur after prolonged therapy, however, it is important to be vigilant for such complications. MRI is more necessary for those patients who have had good chelation therapy but who are at risk of chelation inadequacy with respect to the heart and for those who have had treatment modification in order to follow the efficacy of the changed chelation regime.

\section{Additional factors}

All the discussed mechanisms contributing to the pathogenesis of heart injury should be taken into consideration. The possible prevention of infections to which thalassaemia patients are susceptible as well as awareness and early treatment of these, hormone replacement where there are insufficiencies, control of glucose and calcium should also be taken into consideration. Efforts should be done to maintain pre transfusion $\mathrm{Hb}$ levels close to $10 \mathrm{~g} / \mathrm{dl}$ to reduce as much as reasonable the high $\mathrm{CO}$ state.

Regarding the question of the strength of exercise, patients should avoid isometric exercise and extreme sport, while regular isotonic exercise, manly walking and swimming, should be suggested in patients with adequate cardiac function.

\section{Conclusions}

Heart involvement in TM mainly results from the two contributing factors with diverse action - iron load and high CO state. The recommendation that pre transfusion $\mathrm{Hb}$ should be maintained close to 10 $\mathrm{g} / \mathrm{dl}$ seems a reasonable target for reducing the increased CO. The heart iron load can be accurately and reproducibly estimated only by magnetic resonance imaging while all the formerly used conventional tests, although still valuable in themselves, are limited in their ability to predict the individual patient's cardiac risk.

\section{References}

1. Modell B, Khan M, Darlison M. Survival in $\beta$-thalassemia major in the UK: data from the UK Thalassemia Register. Lancet. 2000;355:2051-2052.

2. Ladis V, Chouliaras G, Berdousi H, Kanavakis E, Kattamis C. Longitudinal study of survival and causes of death in patients with thalassemia major in Greece.Ann N Y Acad Sci.

3. Borgna-Pignatti C, Rigolotto S. De Stefano P, et al. Survival and disease complications in Thalassemia Major. Annals NY Academy of Science. 1998;850:227.

4. Borgna-Pignatti C, Rigolotto S, De Stefano P, et al. Survival and complications in patients with thalassemia major treated with transfusion and deferoxamine. Haematologica. 2004;89:1187-1193.

5. Caro JJ, Ward A, Green TC et al. Impact of Thalassemia major on Patients and Their Families. Acta Haematologica 2002;107:150 157.

6. Aessopos A, Farmakis D, Hatziliami A, et al. Cardiac status in well- 
treated patients with thalassemia major. Eur $\mathrm{J}$ Haematol. 2004;73:359-66.

7. Olivieri NF, Nathan DG, MacMillan JH, et al. Survival in medically treated patients with homozygous $\beta$-thalassemia.NEJM. 1994;331:574-8.

8. Aessopos A, Deftereos S, Farmakis D, et al. Cardiovascular adaptation to chronic anemia in the elderly: an echocardiographic study. Clin Invest Med. 2004;27:265-73.

9. Varat MA, Adolph, RJ, Fowler NO. Cardiovascular effect of anemia. Am.Heart J. 1972;83:415.

10. Aessopos A, Farmakis D, Deftereos S, et al. Cardiovascular effects of splenomegaly and splenectomy in $\beta$-thalassemia. Ann Hematol. 2005;84:353-7.

11. Murray JF, Dawson AM, Sherlock S. Circulatory changes in chronic liver diseases. Am J Med 1958;24:358-367.

12. Tsomi K, Karagiorga-Lagana M, Karabatsos F, et al. Arterial elastorrhexis in $\beta$-thalassaemia intermedia, sickle cell thalassaemia and hereditary spherocytosis. Eur J Haematol. 2001;67:135-41.

13. Pepe A, Positano V, Santarelli MF, et al. Multislice multiecho T2* cardiovascular magnetic resonance for detection of the heterogeneous distribution of myocardial iron overload.J Magn Reson Imaging. 2006;23:662-8.

14. Westwood MA, Anderson LJ, Maceira AM, et al. Normalized left ventricular volumes and function in thalassemia major patients with normal myocardial iron.J Magn Reson Imaging. 2007;25:1147-51.

15. Aessopos A, Deftereos S, Tsironi M, et al. Predictive echo-Doppler indices of left ventricular impairment in B-thalassemic patients.Ann Hematol. 2007 Jan 30.

16. Nemeth E, Ganz T: Hepcidin and iron-loading anemias. Haematologica 2006;91:727-32.

17. Papanicolaou G, Tzilianos M, Christakis J, et al. Hepcidin in iron overload disorders. Blood 2005;105:4103-5.

18. Gardenghi S, Marongiu MF, Ramos P, et al. Ineffective erythropoiesis in $\{\beta\}$-thalassemia is characterized by increased iron absorption mediated by down-regulation of hepcidin and up-regulation of ferroportin. Blood 2007 Feb 15 ahead of print.

19. Esposito BP, Breuer W, Sirankapracha P, Pootrakul P, Hershko C, Cabantchik ZI. Labile plasma iron in iron overload: redox activity and susceptibility to chelation. Blood, 2003;102:2670-7.

20. Glickstein H, El RB, Link G, et al. Action of chelators in iron-loaded cardiac cells: Accessibility to intracellular labile iron and functional consequences. Blood. 2006;108:3195-203.

21. Modell and Berdoukas, The Clinical Approach to Thalassaemia. Grune and Stratton, New York, 1984:165-169.

22. Trinder D, Fox C, Vautier G, Olynyk JK. Molecular pathogenesis of iron overload. Gut 2002;51:290-5.

23. Anderson LJ, Holden S, Davis B, et al. Cardiovascular T2* magnetic resonance for the early diagnosis of myocardial iron overload. Eur Heart J. 2001;22:2171.

24. Wood JC, Tyszka M, Carson S, Nelson M, Coates T. Myocardial iron loading in transfusion dependent thalassemia and sickle-cell disease. Blood. 2003;103,5:1934-1936.

25. Aessopos A, Fragodimitri C, Karabatsos F, et al. Cardiac magnetic resonance imaging $\mathrm{R} 2 *$ assessments and analysis of historical parameters in patients with transfusion-dependent thalassemia. Haematologica. 2007;92:131-2.

26. Tanner MA, Galanello R, Dessi C, et al. Myocardial iron loading in patients with thalassemia major on deferoxamine chelation. J Cardiovasc Magn Reson 2006;8:543-7.

27. Economou-Petersen E, Aessopos A, Kladi A, et al. Apolipoprotein E epsilon4 allele as a genetic risk factor for left ventricular failure in homozygous $\beta$-thalassemia. Blood. 1998;92:3455-9.

28. Ferrara M, Matarese SM, Francese M, et al. Role of apolipoprotein $\mathrm{E}$ (APOE) polymorphism on left cardiac failure in homozygous $\beta$ thalassaemic patients.Br J Haematol. 2001;114:959-60.

29. Tsironi M, Deftereos S, Andriopoulos P, Farmakis D, Meletis J, Aessopos A. Reversal of heart failure in thalassemia major by combined chelation therapy: a case report. Eur J Haematol. 2005;74:845.

30. Wu KH, Chang JS, Tsai CVH, Peng CT. Combined therapy with deferiprone and desferrioxamine successfully regresses heart failure in patients with $\beta$-thalassaemia major. Ann of Haematol 2004;83:471-473.

31. Tanner MA, Galanello R, Dessi C, et al. Combined chelation therapy in thalassemia major for the treatment of severe myocardial siderosis with left ventricular dysfunction. J Cardiovasc Magn Reson. 2008;10:12.

32. Tanner MA, Galanello R, Dessi C, et al. A randomized, placebo-controlled, double-blind trial of the effect of combined therapy with deferoxamine and deferiprone on myocardial iron in thalassemia major using cardiovascular magnetic resonance. Circulation. 2007;115:1876-84.

33. Farmakis D, Giakoumis A, Polymeropoulos E, Aessopos A. Pathogenetic aspects of immune deficiency associated with $\beta$-thalassemia. Med Sci Monit 2003;9:19-22.

34. Walker EM, Walker SM: Effects of iron overload on the immune system. Ann Clin Lab Sci 2000;30:354-365

35. Lesic B, Foulon J, Carniel E. Comparison of the effects of deferiprone versus deferoxamine on growth and virulence of yersinia enterocolitica. Antimicrob agents chemother, June 2002,1741-1745.

36. Khimji PL, Miles AA. Microbial iron-chelators and their action on Klebsiella infections in the skin of guinea-pigs. Br. J Exp Pathol, 1978;59:137-47.

37. Walker EM, Walker SM: Effects of iron overload on the immune system. Ann Clin Lab Sci 2000;30:354-365.

38. Kremastinos DT, Flevari P, Spyropoulou M, Vrettou H, Tsiapras D, Stavropoulos-Giokas CG. Association of heart failure in homozygous $\beta$-thalassemia with the major histocompatibility complex. Circulation 1999;100:2074-8.

39. Khimji PL, Miles AA. Microbial iron-chelators and their action on Klebsiella infections in the skin of guinea-pigs. Br. J Exp Pathol,1978;59:137-47.

40. Engle MA, Erlandson M, Smith $\mathrm{CH}$. Late cardiac complications of chronic, refractory anemia with hemochromatosis. Circulation 1964;30:698-705.

41. Kremastinos DT, Tsetsos GA, Tsiapras DP, Karavolias GK, Ladis VA, Kattamis CA. Heart failure in $\beta$ thalassemia: a 5 -year follow-up study. Am J Med. 2001;111:349-54.

42. Cheung YF, Chan GC, Ha SY. Arterial stiffness and endothelial function in patients with $\beta$-thalassemia major. Circulation. 2002 Nov 12;106:2561-6.

43. Hahalis G, Kremastinos DT, Terzis G, et al. Global vasomotor dysfunction and accelerated vascular aging in $\beta$-thalassemia major. Atherosclerosis. 2007 Nov 5.

44. Aessopos A, Farmakis D, Loukopoulos D. Elastic tissue abnormalities resembling pseudoxanthoma elasticum in $\beta$ thalassemia and the sickling syndromes. Blood. 2002;99:30-5.

45. Cheung YF, Chan GC, Ha SY. Effect of deferasirox (ICL670) on arterial function in patients with $\beta$-thalassaemia major. Br J Haematol. 2008;141:728-33.

46. Kato GJ, McGowan V, Machado RF, et al. Lactate dehydrogenase as a biomarker of hemolysis-associated nitric oxide resistance, priapism, leg ulceration, pulmonary hypertension, and death in patients with sickle cell disease. Blood. 2006;107:2279-85.

47. Derchi G, Forni GL, Formisano F, et al. Efficacy and safety of sildenafil in the treatment of severe pulmonary hypertension in patients with hemoglobinopathies. Haematologica. 2005;90:452-8. 
48. Aessopos A, Farmakis D. Pulmonary hypertension in $\beta$-thalassemia. Ann N Y Acad Sci. 2005;1054:342-9.

49. Tsironi M, Korovesis K, Farmakis D, Deftereos S, Aessopos A. Hypocalcemic heart failure in thalassemic patients. Int $\mathrm{J}$ Hematol. 2006;83:314-7.

50. Nienhuis AW. Vitamin C and iron. N Engl J Med. 1981;304:170-1.

51. Aessopos A, Farmakis D, Deftereos S, et al. Thalassemia heart disease: a comparative evaluation of thalassemia major and thalassemia intermedia. Chest. 2005;127:1523-30.

52. Braunwald E, Zipes D, Libby P. Heart Disease, 6th edition, Philadelphia, WB Saunders, 2001.

53. Wasserman AJ, Richardson DW, Baird CL, Wyso EM. Cardiac hemochromatosis simulating constrictive pericarditis. Am.J.Med. 1960;32:316.

54. Aessopos A, Farmakis D, Karagiorga M, Rombos I, Loucopoulos D. Pseudoxanthoma elasticum lesions and cardiac complications as contributing factors for strokes in $\beta$-thalassemia patients. Stroke. 1997;28:2421-4.

55. Forni GL, Derchi G. Typical manifestation of acute congestive heart failure in patients with thalassemia major causing diagnostic delay in the emergency room. The European Journal of Heart Failure 2003;5:607-8.

56. Hahalis G, Manolis AS; Gerasimidou I, et al. Right ventricular diastolic function in $\beta$-thalassemia major: echocardiographic and clinical correlates. Am Heart J. 2001;141:428-34.

57. Hahalis G, Manolis AS, Apostolopoulos D, Alexopoulos D, Vagenakis AG, Zoumbos NC. Right ventricular cardiomyopathy in $\beta$-thalassaemia major. Eur Heart J. 2002;23:147-56.

58. Venti S, Cainelli F, Cesario F. Infections and Thalassaemia. Lancet Infect Dis 2006;6:226-33.

59. Sermsappasuk P, Baek M, Weiss M. Kinetic analysis of myocardial uptake and negative inotropic effect of amiodarone in rat heart. Eur J Pharm Sci. 2006;28:243-8.

60. Grady RW, Berdoukas VA, Rachmielewitz EA, et al. Optimizing chelation therapy: Combining deferiprone and desferrioxamine. Dec 2000. 42nd Annual Meeting of the American Society of Hematology. San Fransisco.

61. Origa R, Bina P, Agus A, et al. Combined therapy with deferiprone and desferrioxamine in thalassemia major. Haematologica 2005;90:1309-1314.

62. Kontoghiorghes GJ. Future chelation monotherapy and combination therapy strategies in thalassemia and other conditions. comparison of deferiprone, deferoxamine, ICL670, GT56-252, L1NAll and starch deferoxamine polymers. Hemoglobin 2006;3:329-47.

63. Tsironi M, Polonifi K, Deftereos S, et al. Transfusional hemosiderosis and combined chelation therapy in sickle thalassemia.Eur $\mathrm{J}$ Haematol. 2005;75:355-8.

64. Farmaki K, Angelopoulos N, Anagnostopoulos G, Gotsis E, Rombopoulos G, Tolis G. Effect of enhanced iron chelation therapy on glucose metabolism in patients with $\beta$-thalassaemia major.Br J Haematol. 2006;134:438-44.

65. Anderson LJ, Westwood MA, Holden S, et al. Myocardial iron clearance during reversal of siderotic cardiomyopathy with intravenous deseferrioxamine:a prospective study using $\mathrm{T} 2 *$ cardiovascular magnetic resonance. Brit. J. Haemat 2004;127:348-355.
66. Tsironi M, Korovesis K, Farmakis D, Deftereos S, Aessopos A. Hypocalcemic heart failure in thalassemic patients.Int $\mathrm{J}$ Hematol. 2006;83:314-7.

67. Chopra D, Janson P, Sawin C. Insensitivity to digoxin associated with hypocalcemia. N Engl J Med 1977;296:917-918.

68. Chapman RW, Hussain MA, Gorman A, et al. Effect of ascorbic acid deficiency on serum ferritin concentration in patients with $\beta$-thalassaemia major and iron overload. J Clin Pathol 1982;35:487-91.

69. Angelucci E, Brittenham GM, McLaren CE, Ripalti M, Baronciani D, Giardini C. Hepatic iron concentration and total body iron stores in Thalassaemia Major. N.Engl.J.Med. 2000;343:327-331.

70. Berdoukas V, Dakin C, Freeman A, Fraser I, Aessopos A, Bohane T. Lack of correlation between iron overload cardiac dysfunction and needle liver biopsy iron concentration. Haematologica. 2005;90:685-6.

71. Aessopos A, Giakoumis A, Fragodimitri C, et al. Correlation of echocardiography parameters with cardiac magnetic resonance imaging in transfusion-dependent thalassaemia major. Eur J Haematol. 2007;78:58-65.

72. Wood JC, Enriquez C, Ghugre N, et al. MRI R2 and R2* mapping accurately estimates hepatic iron concentration in transfusiondependent thalassemia and sickle-cell disease patients. Blood. 2005; 10:1182. Blood 2004;10:3982.

73. Voskaridou E, Douskou M, Terpos E, et al. Magnetic resonance imaging in the evaluation of iron overload in patients with $\beta$ thalassemia and sickle cell disease. BJH. 2004;126:736-742.

74. Ooi GC, Khong PL, Chan GCF, et al. Magnetic resonance screening of iron status in transfusion-dependent $\beta$-thalassemia patients. BJH. 2004;124; 385-390.

75. Olivieri NF, Brittenham GM, Armstrong SAM et al. First prospective randomised trial of the iron chelators Deferiprone (L1) and Deferoxamine. Blood 1995;86:249.

76. Westwood MA, Firmin DN, Gildo M, et al. Intercentre reproducibility of magnetic resonance $\mathrm{T} 2 *$ measurements of myocardial iron in thalassaemi. Int. J Cardiovasc Imaging (US) 2005;21:531-8.

77. Ghugre NR, Enriquez CM, Coates TD, et. al. Improved R2* measurements in myocardial iron overload. J Magn Reson Imaging 2006;23:9-16.

78. Pennell DJ, Berdoukas V, Karagiorga M, et al. Randomized controlled trial of deferiprone or deferoxamine in $\beta$-thalassemia major patients with asymptomatic myocardial siderosis. Blood. 2006;107:3738-44.

79. Peng CT, Chow KC, Chen JH, Chiang YP, Lin TY, Tsai CH. Safety monitoring of cardiac and hepatic systems in $\beta$-thalassemia patients with chelating treatment in Taiwan. Eur $\mathrm{J}$ Haematol 2003;70:392-7.

80. Eleftheriou P, Tanner M, Pennell D, Porter J. Response of Myocardial $\mathrm{T}^{*}$ to oral deferasirox montherapy for 1 year in 29 patients with transfusion-dependent anaemias; A subgroup analysis. European Haematology Association Annual General Meeting, 2006 Abstract 0999.

81. Wood JC, Otto-Duessel M, Gonzalez I, et al. Deferasirox and deferiprone remove cardiac iron in the iron-overloaded gerbil. Transl Res. 2006;148: 272-80. 